\title{
COVID-19 related muscle denervation atrophy
}

\author{
S. Bahouth ${ }^{1} \cdot$ K. Chuang ${ }^{2} \cdot$ L. Olson ${ }^{3} \cdot$ D. Rosenthal ${ }^{1}$ \\ Received: 9 September 2020 / Revised: 14 January 2021 / Accepted: 19 January 2021 / Published online: 31 January 2021 \\ (C) ISS 2021
}

\begin{abstract}
COVID-19 has presented with a variety of manifestations including peripheral neurological symptoms. The most commonly associated peripheral neuropathies described with COVID-19 are Guillain-Barre syndrome and its variants as well as critical illness polyneuropathy. We report in this paper the distinct MRI findings of an unusual case of peripheral neuropathy associated with COVID-19. These findings are similar to those seen in Guillain-Barre syndrome or one of its variants, although differing from the classic condition in certain key clinical and radiological features.
\end{abstract}

Keywords Covid-19 $\cdot$ Peripheral neuropathy $\cdot$ Guillain-Barre syndrome $\cdot$ Critical illness polyneuropathy $\cdot$ MRI

\section{Introduction}

The world-wide pandemic of COVID-19 has revealed that the disease can have a variety of manifestations. Although it is mostly a respiratory disease, many extra-pulmonary consequences have been reported [1]. Ellul et al. recently published a review of the neurological manifestations of COVID. Anosmia and ageusia are common and well-recognized features of the disease. Other, more serious manifestations include central nervous system disease (encephalitis and acute disseminated encephalomyelitis) and cerebrovascular disease (ischemic stroke, intracerebral hemorrhage, and cerebral venous thrombosis) [2]. Peripheral nervous system disease (Guillain-Barre syndrome, and Guillain-Barre syndrome variants) has also been recognized [2]. However, the proportion of patients with peripheral neurologic symptoms remains low when compared to the pulmonary and other extra-pulmonary manifestations of the disease.

S. Bahouth

sbahouth@mgh.harvard.edu

1 Division of Musculoskeletal Imaging and Intervention, Department of Radiology, Massachusetts General Hospital and Harvard Medical School, 55 Fruit Street, YAW 6048, Boston, MA 02114, USA

2 Department of Neurology, Massachusetts General Hospital and Harvard Medical School, Boston, MA, USA

3 Department of Internal Medicine, Massachusetts General Hospital and Harvard Medical School, Boston, MA, USA
To the best of our knowledge, imaging findings of the peripheral neurological manifestations of COVID have not yet been reported. We encountered an unusual case of peripheral neuropathy with striking findings on MRI. The clinical and imaging findings of this case are discussed below.

The patient has consented to have this case report published.

\section{Case report}

A 63-year-old male was admitted to the hospital after developing a fever and severe respiratory distress due to Covid-19 infection, diagnosed by PCR. During his 37-day hospitalization, he required transfer to the intensive care unit and was intubated for 22 days. He did not require proning or paralytics. On day 25 (3 days after extubation), the patient was alert and conversant. He was able to move his left foot; however, on hospital day 26, the patient developed acute onset left leg weakness. Although he was able to follow commands and had normal motion of his upper extremities and right leg, he had little or no movement in his left lower extremity. He was discharged 11 days later. During the 4 months that have passed since the onset of weakness, his symptoms have not improved and he has developed paresthesias from his left posterior buttock along the posterolateral thigh, anterior shin, and lateral calf to the bottom of his left foot.

Physical examination performed after discharge over video was notable for the absence of active dorsiflexion, plantarflexion, eversion or inversion of the left ankle, with full 
normal active range of motion on the right ankle and symmetric, normal ability to flex and extend both knees. He ambulated with a left steppage gait.

The laboratory evaluation performed included acute phase reactants (CRP and ESR) which were both high $(136 \mathrm{mg} / \mathrm{L}$ and $134 \mathrm{~mm} / \mathrm{h}$, respectively) and a Creatinine Kinase (CK) test that was low at 24 units/L (reference range: 38 174 units/L). Serological work up also included a positive ANA test and a negative mitochondrial antibody test.

An MRI of the brain was performed the day his symptoms developed. It showed symmetrical-restricted diffusion involving the deep frontal white matter superimposed on subtle relatively symmetrical confluent T2/FLAIR hyperintensity in the periventricular white matter and middle cerebellar peduncles. The differential diagnosis for these findings was thought to include toxic-metabolic encephalopathy, delayed post hypoxic leukoencephalopathy or hypoxic ischemic injury. It was not thought to explain his unilateral symptoms. Lumbar spine MRI, also done during his hospital stay, demonstrated no acute findings. Only expected stable diffuse degenerative changes with moderate to severe spinal canal stenosis at L4-L5 were noted.
Four months after his discharge, or roughly $41 / 3$ months after onset of symptoms, a left thigh MRI and a lumbosacral plexus MRI revealed multifocal muscular edema of the thigh. The affected muscles included: the distal and lateral portion of bilateral gluteus maximus (Fig. 1a, b), the left gluteus medius and minimus (Fig. 1a, b), the left biceps femoris, the left semimembranosus and semitendinosus (Fig. 1d), left obturator internus, left quadratus femoris (Fig. 1c), and to a lesser extent the muscles of the left anterior compartment including the vastus lateralis and medialis (Fig. 1c). The left sartorius and the gracilis were not affected. In addition, mild focal thickening of the sciatic nerve on the left at the level of the posterior acetabular column was noted (Fig. 2). The sacral plexus and sciatic nerve proximal and distal to this level had a normal appearance, symmetric to the contralateral side. The upper right thigh was partially included in the lumbar spine examination and appeared normal apart from the mild edema of the gluteus maximus.

His post-discharge work-up also included an EMG that revealed bilateral, generalized, length-dependent, mixed sensorimotor axonal polyneuropathy with superimposed severe left sided nerve conduction and needle electromyography
Fig. $1(\mathbf{a}, \mathbf{b}, \mathbf{c}, \mathbf{d}, \mathbf{e})$ Axial T2 fatsaturated (a) and axial Proton Density (b) images through the pelvis demonstrate extensive muscular edema and fatty atrophy involving bilateral gluteus maximus (red arrows), left gluteus medius (yellow arrow), and left gluteus minimus (green arrow) muscles. Axial T2 fat-saturated image of the pelvis (c) shows left obturator internus (green arrow) and left quadratus femoris (yellow arrow) muscle edema. Axial T2 fat-saturated (d) and axial Proton Density (e) images through the left thigh reveal muscular edema and fatty atrophy of the left biceps femoris (green arrow) and left the semimembranosus and semitendinosus muscles (red arrow)
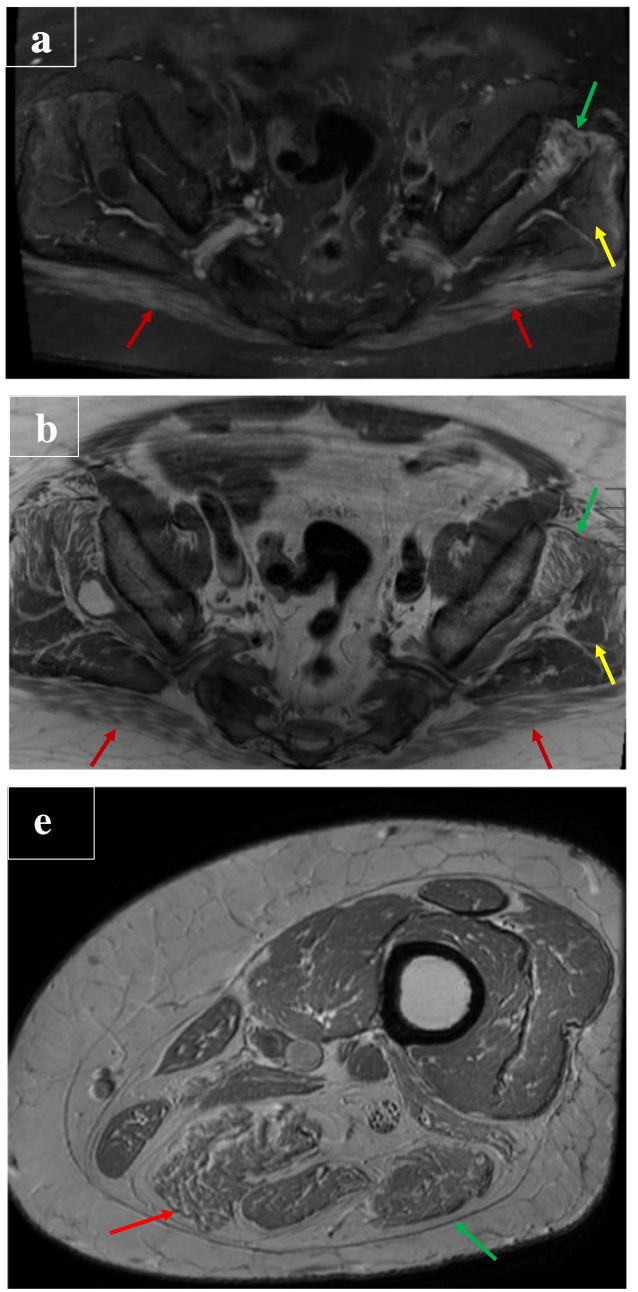
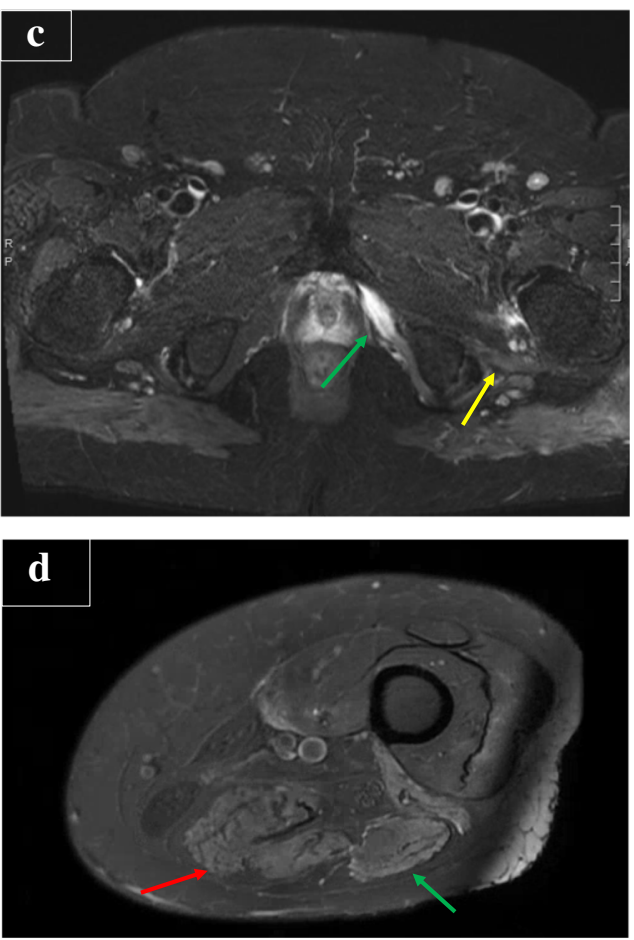


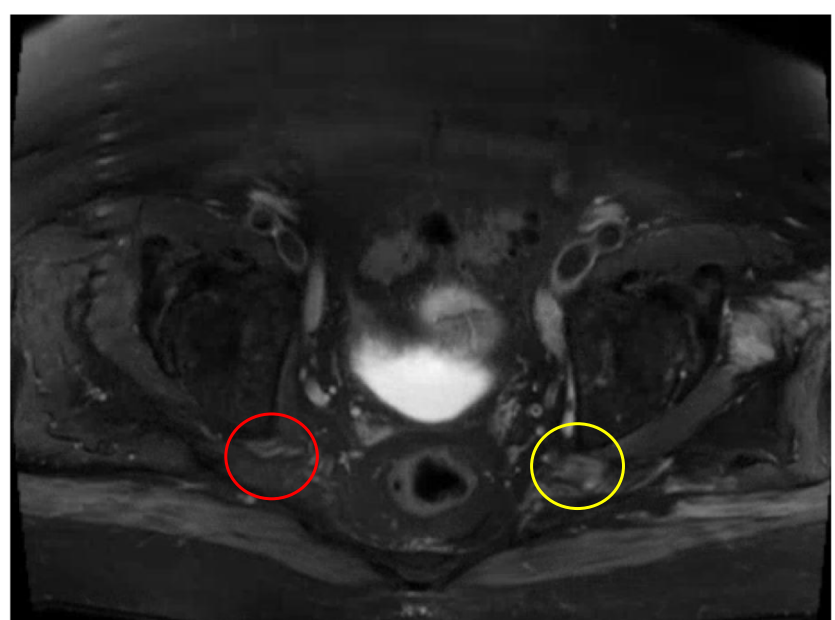

Fig. 2 Axial STIR image through the pelvis show asymmetric left focal thickening and increased signal of the sciatic nerve at the level of the posterior acetabular column (yellow circle). The right sciatic nerve appears normal (red circle) abnormalities suggestive of a left sided sciatic neuropathy or lumbosacral plexopathy. No myopathic units were seen.

Since that time, the patient has been followed clinically. His condition remains unchanged 9 months after symptom onset. No further imaging has been done.

\section{Discussion}

The MRI features of peripheral neuropathies are non-specific, consisting of muscle edema with little or no mass effect in the acute phase, followed by atrophy and fatty replacement when the disease enters a chronic state. The diagnosis can be made most readily when the distribution of findings can be attributed to known patterns of innervation, especially when it is possible to demonstrate abnormalities in the nerve or in the tissues surrounding a nerve.

Our patient's MRI showed multifocal muscular edema in a pattern that cannot be explained as an isolated sciatic

Table 1 Left hip and thigh muscle involvement and respective innervation

\begin{tabular}{|c|c|c|c|c|}
\hline & & $\begin{array}{l}\text { Muscular } \\
\text { edema }\end{array}$ & Nerve & Roots \\
\hline \multirow[t]{12}{*}{ Thigh } & Adductor magnus & Yes & Posterior division of the obturator nerve; tibial nerve & L2-L3-L4-L5 \\
\hline & Adductor longus & No & obturator & L2-L3-L4 \\
\hline & Adductor brevis & No & obturator & L2-L3-L4 \\
\hline & Gracilis & No & obturator & L2-L3 \\
\hline & Biceps femoris & Yes & $\begin{array}{l}\text { Long head: tibial nerve } \\
\text { Short head: peroneal nerve }\end{array}$ & L4-S3 \\
\hline & Semitendinosus & Yes & Tibial nerve & L4-S3 \\
\hline & Semimembranosus & Yes & Tibial nerve & L4-S3 \\
\hline & Sartorius & No & Femoral nerve & L2-L3-L4 \\
\hline & Vastus lateralis & Less & Femoral nerve & L2-L3-L4 \\
\hline & Vastus medialis & Less & Femoral nerve & L2-L3-L4 \\
\hline & Vastus intermedius & Less & Femoral nerve & L2-L3-L4 \\
\hline & Rectus femoris & No & Femoral nerve & L2-L3-L4 \\
\hline \multirow[t]{13}{*}{ HIP } & Gluteus maximus & Yes & Inferior gluteal nerve & $\begin{array}{l}\text { Dorsal branches of the ventral rami of } \\
\text { L5-S1-S2 }\end{array}$ \\
\hline & Gluteus medius & Yes & Superior gluteal nerve & Ventral divisions of L4-L5 and S1 \\
\hline & Gluteus minimus & Yes & Superior gluteal nerve & Ventral divisions of L4-L5 and S1 \\
\hline & Tensor fascia lata & Yes & Superior gluteal nerve & Ventral divisions of L4-L5 and S1 \\
\hline & Pectineus & No & Femoral nerve and sometimes obturator & L2-L4 \\
\hline & Quadratus femoris & Yes & Nerve to quadratus femoris & L4-S1 \\
\hline & Iliacus & Yes & Femoral & L2-L3-L4 \\
\hline & Psoas & Yes & $\begin{array}{l}\text { Direct branches of the anterior rami off the lumbar } \\
\text { plexus }\end{array}$ & L1-L3 \\
\hline & Gemellus superior & Yes & Nerve to obturator internus & L5-S2 \\
\hline & Gemellus inferior & Yes & Nerve to quadratus femoris & L4-S1 \\
\hline & Obturator externus & No & Posterior branch of the obturator nerve & L5-S1-S2 \\
\hline & Obturator internus & Yes & Nerve to the obturator internus $\mathrm{m}$ & Anterior divisions of the sacral plexus L5-S2 \\
\hline & Piriformis & No & Nerve to pyriformis: ventral rami of S1-S2 & $\mathrm{S} 1-\mathrm{S} 2$ \\
\hline
\end{tabular}




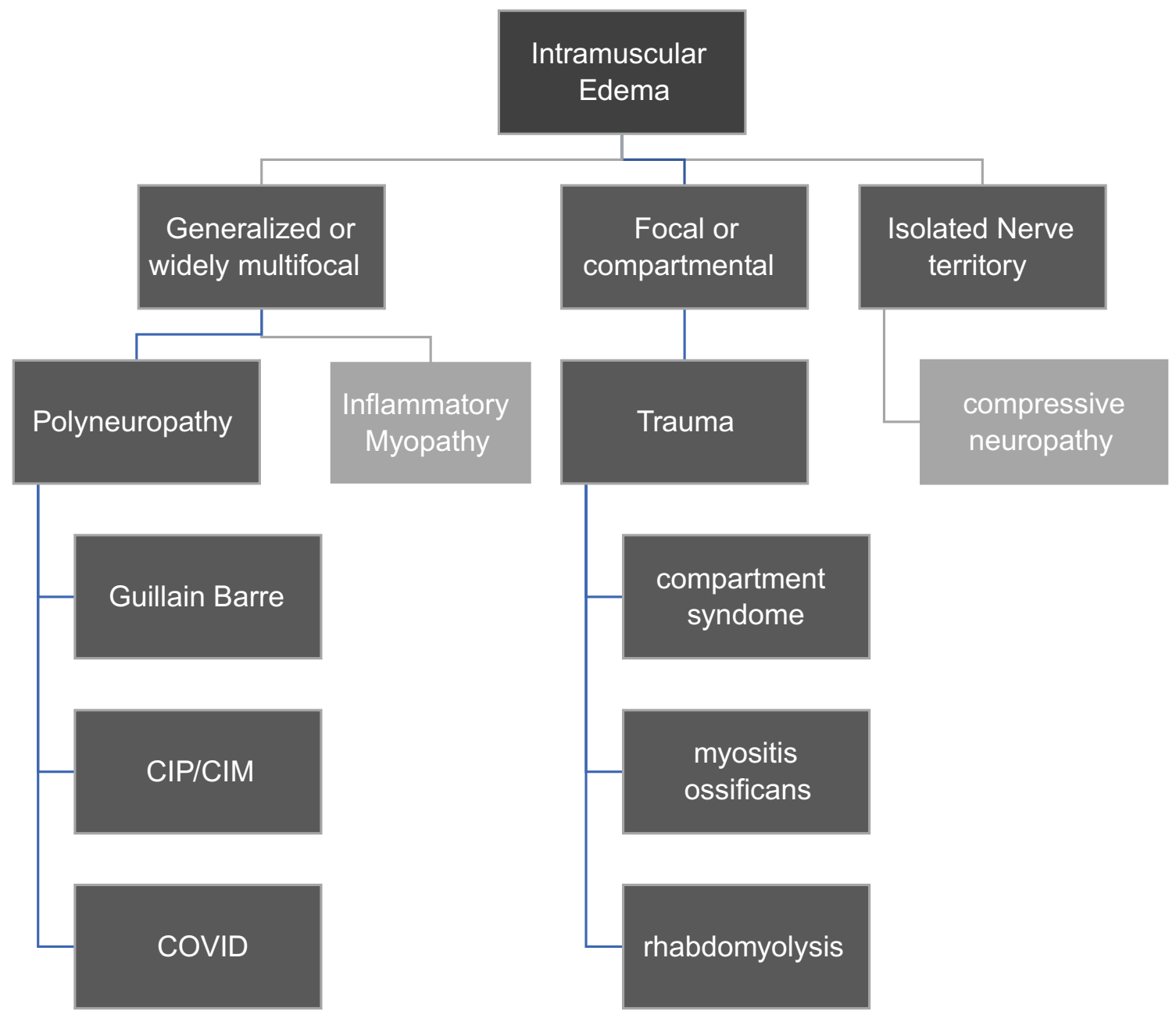

Fig. 3 A limited flowchart illustrating the approach to differential diagnosis of muscle edema, as seen in this case. Not all possibilities can be included in such a format. For example, radiation-related changes (which could be regarded as a form of trauma) are not included, nor is peritumoral edema

mononeuropathy, since muscles innervated by multiple other nerves including the femoral nerve were also affected (Table 1). The main affected muscles included the distal and lateral portion of the gluteus maximus, the biceps femoris, and the semimembranosus and semitendinosus muscles. The muscles of the anterior compartment including the vastus lateralis and medialis were less affected.

Despite involvement of multiple nerve territories, the high T2 signal abnormalities seen in the muscles of our patient's left leg are most likely to be due to nerve pathology. The widespread involvement of muscles in both the anterior and posterior thigh cannot be explained on the basis of direct trauma, and the onset was spontaneous. There was no evidence of any mass effect, and the unilateral monomelic presentation excludes metabolic causes.

Axonal polyneuropathies are thought to develop in the context of Covid-19 as either a direct effect of the virus on the nervous system or an indirect immune mediated, or para- infectious disease [2]. Guillain-Barre syndrome and critical illness polyneuropathy/myopathy (CIP/CIM) are the two main peripheral neuropathies described with Covid-19 in the literature.

Guillain-Barre syndrome is an immune-mediated polyneuropathy, which can be post-infectious in etiology. It typically has an acute or subacute onset. Pain and weakness develop over a few hours or a few weeks. Typically, both sides of the body are affected. It can be life-threatening if respiration is impaired and recovery can be very prolonged.

A number of subtypes are recognized, including isolated motor weakness with or without sensory abnormalities, weakness limited to the pharyngeal muscles, and the Miller Fisher syndrome in which there is ataxia, ophthalmoplegia, and areflexia but no limb weakness.

The clinical presentation of our patient does not completely fit classical Guillain-Barre syndrome or its variants. Although EMG indicated bilateral abnormalities, the patient presented 
with a chronic non-progressive, unilateral left sided leg weakness. The lack of demyelinating features on EMG is also atypical.

Critical illness polyneuropathy (CIP) and critical illness myopathy (CIM) are overlapping syndromes that also must be considered in this case. In CIP and CIM, limb involvement is expected to be symmetrical and generalized. CIP/CIM can resemble Guillain-Barre syndrome. The major clinical difference is that critical illness polyneuropathy usually occurs during an intensive care stay, whereas the onset of Guillain-Barré syndrome is delayed, presenting in the outpatient setting, although it may lead to intensive care unit admission if it is severe [3].

The delayed onset of asymmetrical multifocal, left sided symptoms does not support CIP/CIM as the sole diagnosis, given that CIP/CIM EMG findings are usually symmetrical and generalized. To the best of our knowledge, MRI findings in CIP/CIM have not been reported.

Other differential considerations that might be raised by the observation of multifocal muscle edema on MRI are an inflammatory myopathy, such as polymyositis or rhabdomyolysis. However, clinically, the extremely rapid onset (overnight) is not consistent with an inflammatory myopathy and the lack of pain makes rhabdomyolysis unlikely. In addition, in untreated patients with active inflammatory myopathy or rhabdomyolysis, CK is usually more than 10-fold the upper limit of normal (at least 2000 to 3000 units/L). The MRI features are also not supportive, as the myofascial pattern of edema that is classically described with inflammatory myopathies was not seen [4]. The absence of muscle enlargement accompanying muscle edema on MRI also goes against rhabdomyolysis.

Direct compression could result in similar findings in individual muscles on MRI. However the pattern would be extremely unusual since deep muscles (such as the obturator internus) and the anterior parts of other muscles (the gluteus medius) were affected. Myositis ossificans can affect large volumes of muscle, but it is usually a self-limited process that terminates with ossification. It tends to be limited to one muscle or one compartment. Bilateral forms can occur in patients with severe central nervous system injury, but that was not the case with our patient.

Figure 3 is a flowchart that summarizes the major MRI findings differentiating between the aforementioned diagnoses. It also explains our diagnostic approach from a radiologic perspective.

In this paper, we have reported the first case, to our knowledge, of imaging findings in a patient with COVID-19 neuromuscular disease. The findings are probably best categorized as Guillain-Barre syndrome or one of its variants, although differing from the classic condition in certain key features. The MRI features of COVID neuromuscular disease consist of multifocal asymmetrical muscle edema not confined to a single nerve territory.

\section{References}

1. Gupta A, Madhavan MV, Sehgal K, Nair N, Mahajan S, Sehrawat TS, et al. Extrapulmonary manifestations of COVID-19. Nat Med. 2020;26(7):1017-32. https://doi.org/10.1038/s41591-020-0968-3.

2. Ellul MA, Benjamin L, Singh B, Lant S, Michael BD, Easton A, et al. Neurological associations of COVID-19. Lancet Neurol. 2020;19(9): 767-83. https://doi.org/10.1016/S1474-4422(20)30221-0.

3. Zhou C, Wu L, Ni F, Ji W, Wu J, Zhang H. Critical illness polyneuropathy and myopathy: a systematic review. Neural Regen Res. 2014;9(1):101-10. https://doi.org/10.4103/1673-5374.125337.

4. Garcia J. MRI in inflammatory myopathies. Skelet Radiol. 2000;29(8):425-38. https://doi.org/10.1007/s002560000238.

Publisher's Note Springer Nature remains neutral with regard to jurisdictional claims in published maps and institutional affiliations. 